\title{
Therapie des Rektumkarzinoms
}

\author{
Interdisziplinäre Leitlinie der Deutschen Krebsgesellschaft und ihrer \\ Arbeitsgemeinschaften, der Deutschen Gesellschaft für Chirurgie und der \\ Deutschen Gesellschaft für Verdauungs- und Stoffwechselkrankheiten
}

Als Rektumkarzinome gelten Tumoren, deren aboraler Rand bei der Messung mit dem starren Rektoskop $16 \mathrm{~cm}$ oder weniger von der Anocutanlinie entfernt ist (Internationales Dokumentationssystem für das kolorektale Karzinom 1991 [1]). Die Therapie des Rektumkarzinoms sollte grundsätzlich auf der Basis einer histologischen Untersuchung geplant werden. Neben der prätherapeutischen Sicherung der Karzinomdiagnose ist eine Tumorklassifikation nach den Richtlinien der WHO anzustreben.

\section{Präoperative Diagnostik}

Notwendige Untersuchungen

- Anamnese und klinische Untersuchung (einschließlich digitalrektaler Untersuchung)

- Rektoskopie mit Biopsie

- Koloskopie des gesamten Kolons oder Doppelkontrasteinlauf. Bei hochgradig stenosiertem Rektumkarzinom empfiehlt es sich, den restlichen Darm intraoperativ palpatorisch und koloskopisch innerhalb von 3 Monaten postoperativ abzuklären

- Sonographie des Abdomens

- Röntgen-Thorax in 2 Ebenen

- Tumormarker CEA

- Urinsediment.

Im Einzelfall nützliche Untersuchungen

- Endosonographie obligat vor lokaler Exzision (s. unten).

- Spiral-Computertomographie Abdomen/Becken bei unklarem sonographischen Befund und Verdacht auf organüberschreitendes Tumorwachsum.

- Kernspintomographie als Alternative oder Ergänzung zur Spiral-Computertomographie.

- Spiral-Computertomographie bei sonographischem Verdacht auf Infiltration der Harnwege und/oder Erythrozyturie.

- Zystoskopie bei Verdacht auf Blaseninfiltration.
- Gynäkologische Untersuchung bei Verdacht auf Infiltration von Vagina, Uterus und Adnexe.

- Sphinktermanometrie vor intersphinktärer oder koloanaler Anastomose.

Präoperative histologische Diagnostik

a. Biopsie des Primärtumors

Bei Diagnose eines Karzinoms sollten - soweit möglich - auch Aussagen zum Tumortyp, zum Differenzierungsgrad und zu einer möglicherweise vorhandenen Lymphgefäßinvasion gemacht werden. Für die Bestimmung des Tumortyps ist die WHO-Klassifikation [2] maßgeblich, die zwischen Adenokarzinomen und muzinösen Adenokarzinomen sowie weiteren selteneren Karzinomtypen (z. B. Siegelringzellkarzinom, Plattenepithelkarzinom) unterscheidet.

Für die Bestimmung des histologischen Differenzierungsgrades ist die Unterscheidung sog. «Low-grade-Karzinome» und «High-grade-Karzinome» für die Therapieplanung ausreichend. Als «High-grade-Karzinome» werden schlecht differenzierte, muzinöse und nicht muzinöse Adenokarzinome (G3), Siegelringzellkarzinome, kleinzellige und undifferenzierte Karzinome klassifiziert. Bei Vorliegen eines «High-gradeKarzinoms « empfiehlt es sich, einen größeren Sicherheitsabstand nach distal $(2-3 \mathrm{~cm}$ am frischen Resektat ohne Zug gemessen) einzuhalten [3]. Die intratumorale Heterogenität insbesondere größerer Karzinome kann Ursache dafür sein, daß die «High-grade»-Qualität eines Tumors in einer Biopsie nicht erfaßt wird. Die Diagnose «High-grade-Karzinom» schließt ebenso wie der Nachweis einer Lymphgefäßinvasion lokale Therapiemaßnahmen in kurativer Intention aus $[4,5]$.

\section{b. Polypektomie und lokale Tumorexzision}

Bei ektomierten Adenomen bzw. lokalen Tumorexzisionen ist außer der histopathologischen Dignitätsbeurteilung (Adenom versus Karzinom) auch die Vollständigkeit der Tumorentfernung histologisch zu verifizieren: Entfernung im Gesunden, Entfernung nicht im Gesunden, Entfernung zweifelhaft im

\begin{tabular}{ll}
\hline KARGER & @ 1999 S. Karger GmbH, Freiburg \\
Fax +49 7614520714 & Accessible online at: \\
$\begin{array}{l}\text { E-mail kargergmbh@aol.com } \\
\text { www.karger.com }\end{array}$ & http://BioMedNet.com/karger
\end{tabular}


Gesunden. Letztere Beurteilung sollte dann abgegeben werden, wenn Tumorgewebe im Bereich der durch die Diathermieschlinge verursachten Koagulationsnekrose liegt oder die Abtragungsfläche mangels einer Markierung durch den Endoskopiker nicht identifiziert werden kann [4]. Zur exakten pathologischen Beurteilung muß die Abtragungsfläche mit Tusche, Tipp-Ex oder einer Nadel markiert werden.

Liegt histologisch ein Karzinom vor, so ist darüber hinaus der histologische Tumortyp, der Differenzierungsgrad sowie eine möglicherweise vorhandene Lymphgefäßinvasion $\mathrm{zu}$ dokumentieren. Dabei wird in gleicher Weise wie oben beschrieben zwischen «High-grade»- und «Low-grade-Karzinomen» unterschieden.

Eine Indikation zur radikalen Resektion ergibt sich a) bei Unvollständigkeit der Karzinomentfernung, b) bei «Highgrade-Karzinomen» $(\mathrm{G} 3, \mathrm{G} 4), \mathrm{c})$ bei nachweisbarer Lymphgefäßinvasion und d) bei Invasion der Muscularis propria (pT2), wobei jedoch das individuelle Operationsrisiko einer radikalen Resektion zu berücksichtigen ist [5].

\section{Präoperative (neoadjuvante) Tumortherapie (Konsensus CAO, AIO, ARO 1998 [6]}

Bei T4-Tumoren wird eine präoperative Radiotherapie (vermutlich günstigere Ergebnisse durch präoperative RadioChemotherapie) dann empfohlen, wenn aufgrund des präoperativen Stagings oder nach explorativer Laparotomie eine R0-Resektion nicht erreichbar erscheint. (Mögliches Therapieschema siehe Anlage 1.)

\section{Operative Therapie mit kurativem Ziel}

Eine kurative Therapie des Rektumkarzinoms erfolgt in der Regel durch Resektion des tumortragenden Rektums im Gesunden und En-bloc-Entfernung des regionären Lymphabflußgebietes (sog. radikale Resektion nach internationalem Dokumentationssystem für das kolorektale Karzinom 1991 [1]. In streng selektionierten Fällen (s. unten) ist eine kurative Behandlung auch durch lokale endoskopische mikrochirurgische oder chirurgische Tumorexzision (Vollwandresektion) möglich.

Folgende Operationsverfahren sind bei Einhaltung der Kriterien der onkologischen Chirurgie (s. unten) als gleichwertig anzusehen, wobei die Indikationsstellung von der Tumorlokalisation, der Tumorgröße und anderen Faktoren abhängig ist. Nach Möglichkeit sind kontinenzerhaltende Verfahren zu bevorzugen:

- die anteriore Rektumresektion;

- die abdomino-perineale Rektumexstirpation;

- die intersphinktäre Rektumresektion (auch als abdominoperanale Rektumresektion bezeichnet). Diese Operation setzt besondere Erfahrung voraus.

Die Operation in kurativer Absicht beinhaltet:

- die Absetzung der A. mesenterica inferior zumindest unmittelbar distal des Abgangs der A.colica sinistra;

- die komplette (nicht stumpfe) Entfernung des Mesorektum bei Karzinomen der unteren beiden Rektumdrittel;
- die Einhaltung eines angemessenen Sicherheitsabstandes (s. unten);

- in der Regel die En-bloc-Resektion von tumoradhärenten Organen (multiviszerale Resektion);

- möglichst die Erhaltung der autonomen Nervenstränge (Plexus hypogastricus, Plexus pudendus).

Bei Karzinomen des oberen Rektumdrittels ist ein aboraler Sicherheitsabstand von $5 \mathrm{~cm}$ in situ erforderlich. Dies entspricht am frischen, nicht gespannten Präparat einem Abstand von $3 \mathrm{~cm}$. Die Sicherheitszone ergibt sich aus der Zielsetzung, das dem Rektum anhaftende Fettgewebe (Mesorektum) bis zur Durchtrennungsebene vollständig und nicht konusförmig zu entfernen. Jenseits dieser Sicherheitszone sind keine Satellitenmetastasen im Mesorektum zu erwarten. Bei Karzinomen der unteren beiden Drittel des Rektums sollte das Mesorektum komplett bis zur Puborektalisschlinge entfernt werden. Die Rektumwand wird im Fall einer Anastomosierung nahe der Puborektalisschlinge durchtrennt. Ein aboraler Sicherheitsabstand an der Rektumwand von $2 \mathrm{~cm}$ in situ (entsprechend etwa $1 \mathrm{~cm}$ am frischen, nicht ausgespannten Resektat) ist im allgemeinen ausreichend.

\section{Lokale chirurgische Tumorexzision}

Eine lokale chirurgische Tumorexzision bei Rektumkarzinom (Vollwandexzision) ist unter kurativer Zielsetzung vertretbar bei pT1, «Low-risk-Karzinom» (gute bis mäßige Differenzierung, G1-2, keine Lymphgefäßinvasion). Dabei muß die Entfernung im Gesunden erfolgen (s. oben). Geeignete Operationsmethoden sind die transanale chirurgische Exzision (bei nahe der Kryptenregion gelegenen Tumoren) oder die endoskopische mikrochirurgische Tumorabtragung.

Präoperative Hinweiskriterien für eine lokale Exzision sind:

- Tumordurchmesser unter $3 \mathrm{~cm}$;

- Palpation: Mason Clinical Stage I;

- Endosonographie: uT1 und N0 (Fehlen von metastasenverdächtigen pararektalen Lymphknoten);

- Histologie: gute bis mäßige Differenzierung $(\mathrm{G} 1,2)$, keine Lymphgefäßinfiltration («Low-risk-Karzinom»).

\section{Endoskopische Polypektomie}

In sorgfältig ausgewählten Fällen kann ein frühes Rektumkarzinom auch durch endoskopische Polypektomie kurativ behandelt werden. Näheres siehe Abschnitt Polypektomie bei «Präoperative histologische Diagnostik».

\section{Sondersituationen}

Bei Mehrfachkarzinomen im Kolorektum (synchron 2 oder mehrere Primärtumoren) richtet sich das Resektionsausmaß nach der Lokalisation der Tumoren; für zusätzliche Kolonkarzinome gelten die Empfehlungen zur Behandlung des Kolonkarzinoms.

Beim Rektumkarzinom auf dem Boden einer Colitis ulcerosa oder familiären adenomatösen Polypose ist die Proktokolektomie, soweit möglich unter Erhaltung der Kontinenz, indiziert. Die Karzinomerkrankung, insbesondere in begrenztem Stadium, ist keine Kontraindikation für die Anlage eines ileoanalen Pouch. 
Die Ergebnisse der laparoskopischen Tumorresektion sind derzeit nicht abschließend zu beurteilen, so daß dieses Verfahren nur im Rahmen qualifizierter Studien mit langfristiger Verlaufsbeobachtung zur Anwendung kommen sollte.

Bei Notfallsituationen (Ileus, Tumorperforation, Darmperforation bei stenosiertem Tumor) ist das Vorgehen abhängig von der vorliegenden Situation. Nach Möglichkeit sollen die onkologischen Erfordernisse eingehalten werden.

Die Resektion von (syn- und metachronen) Fernmetastasen (Leber, Lunge, u. a.) ist indiziert, sofern eine Resektion, die den onkologischen Radikalitätsansprüchen benügt (R0-Resektion), erzielt werden kann und das Risiko des Eingriffs vertretbar ist. Bei Inoperabilität siehe unter «Palliativmaßnahmen«.

Bei einem lokoregionalen Tumorrezidiv ist der Versuch der kompletten Tumorentfernung gerechtfertigt. Bei Inoperabilität siehe unter «Palliativmaßnahmen«.

\section{Intraoperative patho-histologische Diagnostik}

Eine intraoperative Schnellschnittuntersuchung der Resektionslinien kann unter der Frage «Resektion im Gesunden?» durchgeführt werden. Eine Dignitätsbestimmung im Schnellschnitt (z.B. bei großen villösen Tumoren) ist aus untersuchungstechnischen Gründen nicht sinnvoll. Biopsien aus der Gegend einer verdächtigen Tumorinfiltration sind zu vermeiden, da diese zu einer lokoregionalen Tumorzelldissemination mit dem Risiko des lokoregionalen Rezidivs führen können (siehe unter «Multiviszerale Resektion»).

\section{Postoperative patho-histologische Diagnostik}

Nach radikaler Tumorresektion sind für die weitere Therapieplanung Aussagen über die lokoregionäre Vollständigkeit der Tumorentfernung (R-Klassifikation), die Invasionstiefe des Tumors (pT-Klassifikation) und den Lymphknotenstatus (pN-Klassifikation) notwendig, wobei sich hieraus unter Umständen die Indikation zur Nachbehandlung mit einer Chemo- bzw. Radiochemotherapie ergibt [2]. Erforderlich ist eine Aussage über die Anzahl untersuchter und befallener Lymphknoten. Aus Gründen des Qualitätsmanagements sollte eine Angabe zu Einriß/Einschnitt in oder durch den Tumor sowie Aussagen zu Sicherheitsabständen bzw. Entfernung des Mesorektums erfolgen. Nach neoadjuvanter Therapie ist ein histologisches Regressionsgrading wünschenswert, wobei die Methodik noch nicht standardisiert ist.

\section{Adjuvante Therapie (Konsensus CAO, AIO, ARO 1998 [6]}

4.1 Voraussetzung für die adjuvante Therapie ist die R0Resektion des Primärtumors. Grundlage für die Indikation zur adjuvanten Therapie nach Tumorresektion ist die pathohistologische Untersuchung des Tumorresektats, insbesondere bezüglich Tumorfreiheit des zirkumferentiellen Resektionsrandes am Mesorektum und bezüglich des Lymphknotenstatus. Zur Festlegung der Kategorie pN0 sollen üblicherweise 12 oder mehr regionäre Lymphknoten untersucht werden (UICC 1997 [7]). Immunzytologische Befunde von isolierten
Tumorzellen in Knochenmarkbiopsien oder Lymphknoten sowie zytologische Tumorzellbefunde in Peritonealspülungen sollen für die Indikation zur adjuvanten Therapie außerhalb von Studien nicht berücksichtigt werden.

4.2 Im Hinblick auf eine postoperative Radio-Chemotherapie ist das kleine Becken so zu versorgen, daß die Verlagerung des Dünndarms in das kleine Becken verhindert wird (z. B. Rektumresektion bzw. Netz oder Netzersatz nach abdominoperinealer Exstirpation).

4.3 Für Patienten im UICC-Stadium I oder nach R0-Resektion von Lokalrezidiven oder Fernmetastasen ist eine adjuvante Therapie außerhalb von Studien nicht indiziert.

4.4 Patienten der UICC-Stadien II (pT3-4, pN0, M0) und III (jedes pT, pN1-2, M0) sollten möglichst in kontrollierte Studien eingebracht werden, um auf diese Weise Aufschluß über die optimale adjuvante Therapie zu erhalten.

4.5 Außerhalb von klinischen Studien wird in den Stadien II und III die postoperative Radio-Chemotherapie empfohlen. Diese kann nach der NCI-Empfehlung von 1990/91 erfolgen (siehe Anlage 2). Statt der 5-FU-Bolusapplikation kann auch simultan eine niedrigdosierte 5-FU-Dauerinfusion während der Strahlentherapie (siehe Anlage 2) erfolgen. ${ }^{1}$

4.6 Der Wert der adjuvanten Radiotherapie bei totaler Mesorektumentfernung (TME) ist bisher nicht geklärt.

4.7 Innerhalb der Arbeitsgruppe konnte kein Konsens darüber erzielt werden, ob außerhalb von Studien bei bestehenden Kontraindikationen gegen die Chemotherapie im Rahmen der adjuvanten Radiochemotherapie eine adjuvante Therapie mit dem Antikörper 17-1A zu empfehlen ist.

4.8 Der Verlauf von Patienten, die außerhalb klinischer Studien behandelt werden, ist im Rahmen der Qualitätssicherung hinsichtlich des Auftretens von Rezidiven, der Überlebensrate und von Nebenwirkungen zu dokumentieren.

\section{Radiotherapie}

Die intraoperative Radiotherapie wird derzeit an einigen Zentren überprüft und sollte nur im Rahmen von Studien zur Anwendung kommen.

Eine alleinige Strahlentherapie (endokavitär und/oder perkutan) kann allenfalls bei Inoperabilität des Patienten erwogen werden.

Nach lokoregionär inkompletter Tumorresektion (R1-, R2Resektion) kann der Versuch angezeigt sein, mit einer post-

\footnotetext{
${ }^{1}$ Diese Empfehlung des Konsensus vom Januar 1998 beruht auf einer Mehrheitsmeinung der erstellenden Expertengruppe. Von der Minderheit dieser Expertengruppe wird ebenso wie von den Experten eines Internationalen Symposiums (Soreide, Norstein, Fielding, Silen [8]) die Meinung vertreten, daß bei Einhaltung der in diesen Leitlinien empfohlenen modernen Prinzipien der operativen Therapie mit kurativem Ziel (insbesondere der diesbezüglichen Empfehlungen zum Vorgehen bezüglich Mesorektum) die Indikationen zur adjuvanten Therapie als ungeklärt anzusehen sind und aufgrund entsprechender klinischer Studien neu zu definieren sind. Das Tumorzentrum München hat auch ausführlich dargelegt, daß für die adjuvante Therapie im Stadium II die Datenlage unzureichend ist (Tumorzentrum München 1997 [9], Seiten 53-55, 176-177).
} 
operativen Radiochemotherapie doch noch ein kuratives Therapieziel zu erreichen.

\section{Palliativmaßnahmen}

Zur Palliativbehandlung stehen chirurgische, endoskopische, strahlentherapeutische, chemotherapeutische, medikamentöse und interventionell radiologische Maßnahmen zur Verfügung. Der Einsatz erfolgt individuell abhängig von der vorliegenden Situation. Auch bei Vorliegen von Fernmetastasen kann die operative Entfernung des Primärtumors indiziert sein, wobei neben dem operativen Risiko die Beeinträchtigung der Lebensqualität durch eine Kolostomie bei der Entscheidung zur Operation besondere Berücksichtigung finden muß.

Bei inoperablem lokoregionären Rezidiv ist die Verabreichung einer systemischen Chemotherapie erwiesenermaßen ineffektiv. Gegebenenfalls kann eine Strahlentherapie oder eine Beckenperfusionsbehandlung über perkutane Katheter erwogen werden. Wesentlich ist eine adäquate Schmerztherapie. Bei Unverträglichkeit kommt die Morphinapplikation über einen Periduralkatheter in Betracht.

Bei diffusen nichtresektablen Metastasen sollte die Behandlung bei Nachweis von Metastasierung auf der Basis von 5-FU und Folinsäure begonnen werden. Die Therapie ist zu ändern, wenn die Kontrolluntersuchungen nach 2-3 Zyklen einen Progreß zeigen. Bei stabiler Erkrankung oder bei Ansprechen zu diesem Zeitpunkt sollte die Therapie bis zum nachgewiesenen Progreß fortgeführt werden. Bei Tumorprogression (PD) während oder kurz nach Beendigung der First-line-Therapie wird eine Second-line-Behandlung eingeleitet. Als Secondline-Therapie stehen in Abhängigkeit von der Primärtherapie eine protrahierte Infusion mit 5-FU/FS, eine Behandlung mit CPT-11 oder Oxaliplatin/F-FU/FS zur Verfügung. In Anbetracht der Komplikationsmöglichkeiten der regionalen Chemotherapie kann diese derzeit nicht als Standardverfahren gelten, sondern sollt nur in Studien Anwendung finden. Eine Laparatomie ausschließlich zur Einlage eines Katheters in die A. hepatica ist nur in Ausnahmefällen gerechtfertigt.

\section{Nachsorge (siehe Leitlinien der Deutschen Krebsgesellschaft Nachsorge und Rehabilitation bei Patienten mit gastrointestinalen Tumoren [10])}

Bei frühem Tumorstadium (UICC I) ist nach radikaler R0Resektion in Anbetracht des geringen Rezidivrisikos und der günstigen Prognose von regelmäßigen Nachsorgeuntersuchungen kein Gewinn zu erwarten. Eine Koloskopie nach 2 und 5 Jahren dient der Früherkennung von Zweittumoren (Anlage 3). Abweichend hiervon kann im Einzelfall bei Annahme eines hohen Rezidivrisikos aufgrund des intraoperativen Befundes (z. B. erhöhtes Lokalrezidivrisiko nach intraoperativer Tumoreröffnung) oder eines pathohistologischen Befundes (z.B. erhöhtes Risiko für Lebermetastasen bei Invasion perirektaler Venen oder G3/4-Tumoren) eine regelmäßige oder engmaschige Nachsorge angezeigt sein. Bei Patienten, bei denen eine lokale Tumorexzision durchgeführt bzw. das frühe Karzinom durch endoskopische Polypektomie entfernt wurde, sollten wegen des möglicherweise höheren lokoregionären Rezidivrisikos rektoskopische Untersuchungen eventuell mit Endosonographie in sechsmonatigen $\mathrm{Ab}$ ständen erfolgen (Anlage 4). Nach palliativer Resektion von Rektumkarzinomen sollte die Nachbetreuung symptomorientiert erfolgen.

Regelmäßige Nachuntersuchungen sind zu empfehlen bei Patienten nach R0-Resektion von Tumoren der UICC-Stadien II und III, sofern der Allgemeinzustand und die Lebenserwartung einen Eingriff bei Rezidiv vertretbar erscheinen lassen. (Nachsorgeschema siehe Anlage 5). Tumoren, die nicht eindeutig dem Rektum oder Sigma zuzuordnen sind (sogenannte Rektosigmoidkarzinome), werden in der Tumornachsorge wie Rektumkarzinome behandelt.

\section{Rehabilitation [11]}

Ziel jeder Rehabilitation sind die Sicherung und erforderlichenfalls Verbesserung der Lebensqualität des Betroffenen, wobei die Notwendigkeit rehabilitativer Maßnahmen individuell einzuschätzen ist. Der Rehabilitationsbedarf bei Patienten nach kolorektalen Tumoren ist äußerst variabel und abhängig von Art und Ausmaß des operativen Vorgehens. Rehabilitationsverfahren sollten möglichst im Anschluß an die Primärtherapie stattfinden.

Eine psychosoziale Betreuung und Beratung ist wünschenswert bei Problemen der psychischen Verarbeitung des Tumorleidens, bei aufgetretenen Therapiefolgen (Kontinenzproblemen, sexuellen Funktionsstörungen usw.), bei sozialen Anpassungsstörungen sowie bei der beruflichen Wiedereingliederung.

Gegebenenfalls können stationäre Rehabilitationsverfahren erforderlich sein, um die benötigten Maßnahmen koordiniert und konzentriert einzusetzen. Kontakte zu Selbsthilfeorganisationen (ILCO) sind zu empfehlen.

\section{Anlage 1: Rektumkarzinom T4 [6]. Mögliches Therapieschema bei präoperativer Radio- Chemotherapie.}

1. Bestrahlungsvolumen: Hintere Beckenhälfte von Deckplatte LKW 5 bis Beckenboden, $1 \mathrm{~cm}$ lateral der Linea terminalis.

2. Bestrahlungstechnik: 3- bis 4-Felder-Box, individuell kollimierte Felder, Bestrahlung aller Felder täglich.

3. Bestrahlungsdosis: Einzeldosis 1,8 Gy/Referenzpunkt, 5× wöchentlich, bis 50,4 Gy/Referenzpunkt (Dosismaximum $<55$ Gy). Die 90\%-Isodose umschließt das Zielvolumen. Bei Radio-(Chemo-)Therapie vor abdomino-perinealer Rektumexstirpation können 56 Gy appliziert werden, sofern das Dosismaximum weniger als $5 \%$ höher liegt.

4. Chemotherapie: $1000 \mathrm{mg} 5-\mathrm{FU} / \mathrm{m}^{2} / \mathrm{Tag}$ als Dauerinfusion über 5 Tage in der 1. und 5. (6.) Bestrahlungswoche.

5. Operationszeitpunkt nach Vorbestrahlung: 4 bis 6 Wochen nach Abschluß der Radiotherapie. 


\section{Verfahren zur Konsensbildung}

1) Primär Erstellung durch Expertengruppe der Arbeitsgemeinschaften der Deutschen Krebsgesellschaft:

Chirurgische Arbeitsgemeinschaft für Onkologie (CAO) Arbeitsgemeinschaft für Internistische Onkologie (AIO) Arbeitsgemeinschaft für Radiologische Onkologie (ARO) Arbeitsgemeinschaft für Rehabilitation und Nachsorge (ARNS)

Mitglieder der Expertengruppe waren:

Prof. Dr. med. F.W. Eigler, Essen (CAO)

Prof. Dr. med. H. Gabbert, Düsseldorf (Pathologie)

Prof. Dr. med. Ch. Herfarth, Heidelberg (CAO)

Prof. Dr. med. P. Hermanek, Erlangen

(ISTO, Pathologie)

Prof. Dr. med. W. Hohenberger, Erlangen (CAO)

Prof. Dr. med. D.K. Hossfeld, Hamburg (AIO)

Prof. Dr. med. Th. Junginger, Mainz (CAO)

Dr. med. P. Kruck, Bad Kreuznach (ARNS)

Prof. Dr. med. H.J. Meyer, Solingen (CAO)

Prof. Dr. med. H. Pichlmaier, Köln (CAO)

Prof. Dr. med. R. Sauer, Erlangen (ARO)

Prof. Dr. med. W. Stock, Düsseldorf (CAO)

Beratend haben mitgewirkt:

Prof. Dr. med. H.G. Beger, Ulm (CAO)

Prof. Dr. med. W. Hartel, München (CAO)

Prof. Dr. med. F.W. Schildberg, München (CAO)

Prof. Dr. med. P.M. Schlag, Berlin (CAO)

Arbeitsgemeinschaft Deutscher Tumorzentren (ADT)

Deutsche Gesellschaft für Chirurgie

Deutsche Gesellschaft für Hämatologie und Onkologie

Deutsche Gesellschaft für Innere Medizin

Deutsche Gesellschaft für Pathologie

Deutsche Gesellschaft für Radioonkologie

Deutsche Gesellschaft für Verdauungs- und

Stoffwechselkrankheiten

Deutsche Röntgengesellschaft
2) Überarbeitung mit der Technik des nominalen Gruppenprozesses durch eine von der Deutschen Gesellschaft für Verdauungs- und Stoffwechselkrankheiten (DGVS) unter der Schirmherrschaft der Deutschen Krebsgesellschaft (DKG) am 15. und 16.1.1999 in Bochum veranstaltete Konsensuskonferenz.

Mitglieder der Expertengruppe waren W. Schmiegel (AGO/ DGVS), P. Drings (DKG), S. Petrasch (AGO/DGVS), R. Porschen (AGO/DGVS), H.-J. Schmoll (AIO). Ferner nahmen Vertreter folgender Arbeitsgemeinschaften und Gesellschaften teil:

Arbeitsgemeinschaft Gastroenterologische Onkologie der DGVS (AGO)

Arbeitsgemeinschaft Internistische Onkologie der

DKG (AIO)

Arbeitsgemeinschaft Radiologische Onkologie der

DKG (ARO)

Chirurgische Arbeitsgemeinschaft der DKG und der

Deutschen Gesellschaft für Chirurgie (CAO)

Deutsche Gesellschaft für Chirurgie

Deutsche Gesellschaft für Humangenetik

Deutsche Gesellschaft für Klinische Chemie

Deutsche Gesellschaft für Pathologie

Deutsche Gesellschaft für Radioonkologie

Deutsche Gesellschaft für Verdauungs- und

Stoffwechselkrankheiten

Deutsche Röntgengesellschaft

Koordination:

Prof. Dr. med. Th. Junginger, Mainz (CAO)

Informationszentrum für Standards in der Onkologie (ISTO) bei der Deutschen Krebsgesellschaft, Frankfurt

Korrespondenzadresse:

Klinik und Poliklinik für Allgemein- und Abdominalchirurgie der Johannes-Gutenberg-Universität

Langenbeckstraße 1

D-55101 Mainz

Erstellungsdatum: Februar 1999

Revision geplant: Februar 2000 